\title{
Kikuchis lymphadenitis: Is biopsy necessary in managing these patients
}

Kikuchis-Fujimoto's is a benign, self-limiting, cervical lymphadenitis, fine-needle aspiration cytology (FNAC) plays a very important role in the diagnosis and management of these patients. We present a case of 30-year-old female presenting with supraclavicular lymphadenopathy and fever, diagnosed as Kikuchis-Fujimoto's lymphadenitis on FNAC with no recurrence or development of Lupus lymphadenitis even after 1-year of follow-up. Although Kikuchis-Fujimoto's is a rare cause of lymphadenopathy, FNAC is an important tool in diagnosing this entity and differentiating from tubercular lymphadenitis and Lupus adenitis. Presence of cresentric nucleated histiocytes engulfed karryorrhectic debris and monocytoid cells aids in the diagnosing. A definitive diagnosis made on FNAC can avoid biopsy and unnecessary diagnostic and therapeutic interventions in these patients. However, a look out for recurrence and development of Lupus with regular followup and serological examination is imperative.

Key words: Fine-needle aspiration cytology, kikuchis-fujimoto's, lupus adenitis, tubercular adenitis

\section{INTRODUCTION}

Kikuchis-Fujimoto's disease (KFD) or histiocytic necrotising lymphadenitis was first described by Kikuchis, simultaneously by Fujimoto's et al. in 1972. ${ }^{[1,2]}$ Since then, numerous cases have been reported in both Asian and western population. Though a self-limiting disease that resolves on its own within few weeks and months, it is known to recur in 3-4\% of cases. ${ }^{[3]}$ Though fine-needle aspiration cytology (FNAC) is diagnostic in most of these cases, histopathological examination of the excised node is usually done to confirm the diagnosis and differentiate from other causes of adenitis.

Chinaiah S. B. R. Prasad

Departments of Pathology and ${ }^{1}$ Otorhinolaryngology, Sri Devaraj Urs Medical College, Kolar, Karnataka, India

Address for the Correspondence: Dr. Hemalatha A, Department of Pathology, Sri Devaraj Urs Medical College, Tamaka, Kolar - 563 101, Karnataka, India.

E-mail: drhemashashi@gmail.com

\begin{tabular}{l} 
Access this article online \\
\hline Website: www.ijmedph.org \\
DOI: 10.4103/2230-8598.151275 \\
\hline Quick response code: \\
\hline
\end{tabular}

\section{CASE REPORT}

A 31-year-old female presented with fever (low grade, Intermittent), headache, edema, weight loss and pain in the right supraclavicular region of 3 month's duration. There was no history of cough with expectoration/weight loss/skin rash/joint pain or any other swellings in the body. On examination, she was febrile, with multiple nodules in right supraclavicular region. A diagnosis of viral fever with supraclavicular lymphadenopathy and was made, and she was treated symptomatically. Since her symptoms did not subside even after 1 week of treatment, the presumptive diagnosis of tubercular lymphadenitis was made, and laboratory workup was suggested. All her biochemical and hematological investigations were normal except an increase in erythrocyte sedimentation rate $(80 \mathrm{~mm}$ at the end of $1^{\text {st }} \mathrm{h}$ ). Antinuclear antibody test was negative.

An ultrasonography of right supraclavicular region revealed multiple nodes in upper and middle jugular areas largest measuring $1.4 \mathrm{~cm}$ across. A diagnosis of reactive lymph node enlargement was given.

Fine-needle aspiration cytology of the node was highly cellular with a heterogeneous population of lymphocytes, histiocytes, cresentric nucleated histiocytes [Figure 1] (histiocyte with cresentric nucleus and eosinophilic inclusions in the cytoplasm [Figure 2]), macrophages with engulfed karryorrhectic debris and monocytoid cells. Background showed karryorrhectic debris and erythrocytes. Ziehl Neelson for tubercular acid-fast bacilli stain, periodic acid-Schiff stain, Grams-stain did not reveal any organisms. 


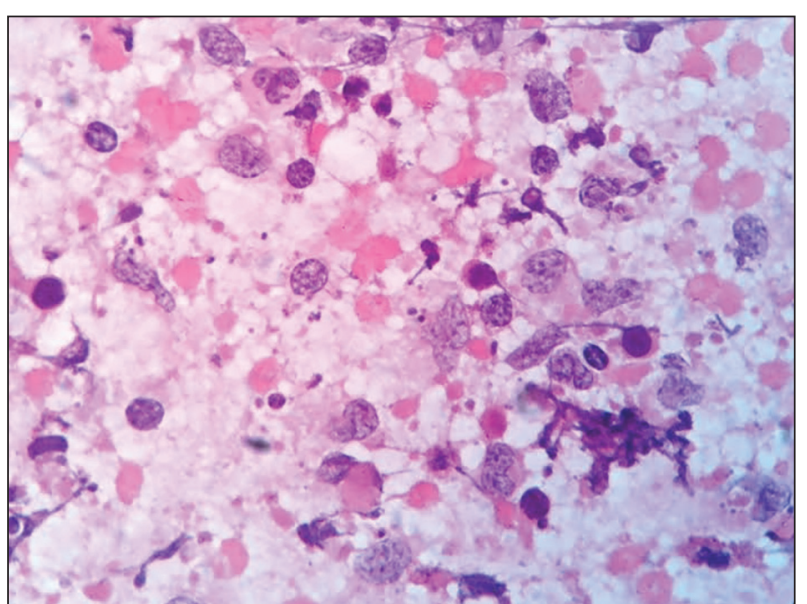

Figure 1: Heterogeneous population of lymphocytes, histiocytes, cresentric nucleated histiocytes in a background of karyorrhectic debris ( $\mathrm{H}$ and $\mathrm{E}, \times 200$ )

A diagnosis of Kikuchis-Fujimoto's Lymphadenitis in necrotizing stage was made. Patient was treated symptomatically along with a low dose of corticosteroid. After 3 weeks of treatment, her swelling subsided and there was no relapse or new symptoms even after 1-year of initial presentation.

\section{DISCUSSION}

Kikuchis Lymphadenitis is a rare, benign, self-limiting, immune mediated disorder occurring in young females that resolves on its own. ${ }^{[4]}$ Though Kikuchis Lymphadenitis can be diagnosed on fine needle aspiration many prefer Lymph node Biopsy for clinching the diagnosis.

Kuo proposed classification of the histopathological features of KFD into three stages-proliferative, necrotizing and xanthomatous stage. The most common stage is the proliferative phase which is characterized by numerous histiocytes, plasmacytoid monocytes, and a variable number of lymphoid cells, karyorrhectic nuclear fragments and eosinophilic apoptotic debris.

If any degree of coagulative necrosis is there, it is necrotic stage, while foamy histiocytes predominate in the xanthomatous stage. He also suggested that these types may suggest different stages of the disease or may reflect differences in cause or host reaction. ${ }^{[4]}$

Fine-needle aspiration findings may vary depending on the stage of the disease. Most common presentation is the stage of necrotizing adenitis wherein presence of cresentric histiocyte (histiocyte with cresentric nucleus and eosinophilic inclusions in the cytoplasm), macrophages with abundant cytoplasm and phagocytosed karyorrhectic or eosinophilia debris, plasmacytoid monocytes were consistent with Kikuchis lymphadenitis.

Though numerous differentials are described, in our country differentials such as such as tuberculosis, Systemic Lupus lymphadenitis are important.

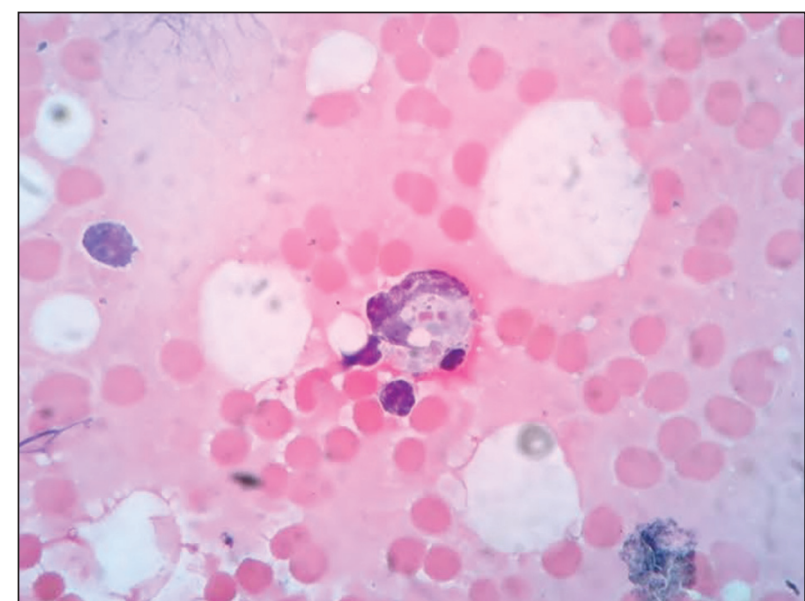

Figure 2: Histiocyte with cresentric nucleus and eosinophilic inclusions in the cytoplasm $(\mathrm{H}$ and $\mathrm{E}, \times 400)$

Tuberculosis can be differentiated by presence of cheesy necrosis, granulomas, epithelioid histiocytes admixed with neutrophils and absence of any acid-fast bacilli on Ziehl Neelson staining. While the presence of cresentric histiocytes might be a sensitive and considerable specific marker of Kikuchis Lymphadenitis. ${ }^{[5]}$

Lupus adenitis can have a varied presentation. Kucukardali et al. reported 28 cases of Lupus associated KL, wherein 18 patients were diagnosed with Lupus and KL simultaneously, four had previous diagnosis of lupus, six were diagnosed with lupus after they were diagnosed with KL. ${ }^{[6]}$

Lupus adenitis can be differentiated by presence of polymorphous population of lymphocytes, Hematoxylin bodies and immunoblasts in Lupus adenitis as compared to Kikuchis adenitis. Absence of neutrophils will favor a diagnosis of Kikuchis lymphadenopathy. However, antinuclear antibodies and antidouble stranded DNA antibody in serum will confirm the diagnosis. ${ }^{[7,8]}$

Although histopathology will help us to confirm the diagnosis, patient will be relieved of the burden of surgery, pain by making a definitive diagnosis on aspiration keeping in mind the above findings in India were tuberculosis is more common. However, a follow-up of these patients is absolutely necessary as these patients are known to have recurrences or known to develop Lupus. ${ }^{[9]}$

\section{CONCLUSION}

Diagnosis of KFD is possible on FNAC. Keeping in mind the above cytological features it is possible to make a definitive diagnosis and avoid biopsy in these patients. However due to a higher incidence of recurrence and occurrence of Lupus in these patients, regular followup and serological testing is important.

\section{REFERENCES}

1. Kikuchis M. Lymphadenitis showing focal reticulum cell hyperplasia with nuclear debris and phagocytosis. Nippon Ketsueki Gakkai Zasshi 1972;35:378-80. 
2. Fujimoto $Y$, Kozima $Y$, Hamaguchi K. Cervical necrotizing lymphadenitis: A new clinicopathological agent. Naika 1972;20:920-7.

3. Rezayat T, Carroll MB, Ramsey BC, Smith A. A case of relapsing KikuchiFujimoto disease. Case Rep Otolaryngol 2013;2013:364795.

4. Kuo T. Kikuchis disease (histiocytic necrotizing lymphadenitis): A clinicopathologic study of 79 cases with an analysis of histologic subtypes, immunohistology and DNA policy. Am J Surg Pathol 1995;19:798-809.

5. Han KM, Go JH, Myong NH, Lee W. Fine needle aspiration cytology of Kikuchi's lymphadenitis: With emphasis on differential diagnosis with tuberculosis. Korean J Pathol 2011;45:626-31. Available from: http://www. dx.doi.org/10.4132/. [Last accessed on 2014 Mar 7].

6. Kucukardali Y, Solmazgul E, Kunter E, Oncul O, Yildirim S, Kaplan M. Kikuchi-Fujimoto Disease: Analysis of 244 cases. Clin Rheumatol 2007;26:50-4.
7. Pai MR, Adhikari P, Coimbatore RV, Ahmed S. Fine needle aspiration cytology in systemic lupus erythematosus lymphadenopathy. A case report. Acta Cytol 2000;44:67-9.

8. Martinez-Vazquez C, Hughes G, Bordon J, Alonso-Alonso J, Anibarro-Garcia A, Redondo-Martinez E, et al. Histiocytic necrotizing lymphadenitis, Kikuchi-Fujimoto's disease, associated with systemic Lupus erythematosus. Q J Med 1997;90:531-3.

9. Patra A, Bhattacharya SK. SLE Developing in a follow-up patient of Kikuchi's disease: A rare disorder. J Clin Diagn Res 2013;7:752-3.

How to cite this article: Anantharamaiah $\mathrm{H}$, Arokiyaswamy $\mathrm{S}$, Prasad CS. Kikuchis lymphadenitis: Is biopsy necessary in managing these patients. Int J Med Public Health 2015;5:115-7. Source of Support: Nil, Conflict of Interest: None declared.

Staying in touch with the journal

1) Table of Contents (TOC) email alert Receive an email alert containing the TOC when a new complete issue of the journal is made available online. To register for TOC alerts go to www.??????/signup.asp.

\section{2) RSS feeds}

Really Simple Syndication (RSS) helps you to get alerts on new publication right on your desktop without going to the journal's website. You need a software (e.g. RSSReader, Feed Demon, FeedReader, My Yahoo!, NewsGator and NewzCrawler) to get advantage of this tool. RSS feeds can also be read through FireFox or Microsoft Outlook 2007. Once any of these small (and mostly free) software is installed, add www.????/rssfeed.asp as one of the feeds. 